\title{
Effective Mass Schrödinger Equation via Point Canonical Transformation
}

\author{
Altu $\operatorname{Arda}^{1, *}$ and Ramazan Sever $^{2,+}$ \\ ${ }^{1}$ Department of Physics Education, Hacettepe University, Ankara 06800,Turkey \\ ${ }^{2}$ Department of Physics, Middle East Technical University, Ankara 06531, Turkey
}

\begin{abstract}
Exact solutions of effective radial Schrödinger equation are obtained for some inverse potentials by using the point canonical transformation. The energy eigenvalues and the corresponding wave functions are calculated by using a set of mass distributions
\end{abstract}

PACS numbers: 03.65.- $03.65 . G e$ 12.39.Fd

*E-mail: arda@hacettepe.edu.tr

${ }^{\dagger}$ E-mail: sever@metu.edu.tr 
In quantum mechanics, exactly solvable problems are suited in the class having shape invariance potential. ${ }^{[1]}$ The potential in a given class can be converted to one another by using the so-called point canonical transformation (PCT). ${ }^{[2]}$ In the PCT approach, which makes it possible to construct a map between the wave equations written for two different potentials, it is needed to know the energy eigenvalues, and the corresponding wave functions of a given potential (reference potential $U(x)$ ) to obtain the energy spectra, and wave functions of the other potential (target potential $V(x)$ ). The PCT has been used by many authors ${ }^{[3-6]}$ to find the energy spectrum, and their wave functions of some potentials in non-relativistic, and relativistic scheme.

Recently, there are some considerable efforts in the literature about the developments of the PCT approach to extend to the case of position-dependent mass (PDM) ${ }^{[2,7-13]}$ and also the PDM formalism has found a wide application area in the literature. ${ }^{[14-18]}$ In this Letter, we choose the potentials $V(\rho)=-\frac{a}{\rho}+c \rho^{p}(p=0,-2)$ and $V(\rho)=\frac{a}{\rho^{2}}+c \rho^{2}$ as the reference potential in the case of the radial effective-mass Schrödinger equation (SE), and find the exact energy eigenvalues, and their wave functions of the target potentials by using the PCT. We follow the approach studied in Ref. [2] where the radial effective mass SE was solved for isotropic oscillator. We try to show that the above mentioned formalism could be used for inversely linear and inversely quadratic potential forms.

The SE is written in the case of PDM $\left(m_{0}=\hbar=1\right)^{[2]}$

$$
\left\{\frac{1}{2 m(x)} \frac{d^{2}}{d x^{2}}-\frac{1}{2 m^{2}(x)} \frac{d m(x)}{d x} \frac{d}{d x}-V(x)\right\} \phi_{n}(x)=-E_{n} \phi_{n}(x),
$$

and in the case of constant mass

$$
\left\{\frac{1}{2} \frac{d^{2}}{d y^{2}}-U(y)\right\} \psi_{n}(y)=-\epsilon_{n} \psi_{n}(y),
$$

where $E_{n}$ and $\phi_{n}(x)$ are the eigenvalues and the eigenfunctions of the target potential, while $\epsilon_{n}$ and $\psi_{n}(y)$ denote the eigenvalues, and the eigenfunctions of the reference potential.

By using the transformations ${ }^{[2]}$

$$
\begin{aligned}
y & =h(x), \\
\psi_{n}(y) & =\pi(x) \phi_{n}(x) .
\end{aligned}
$$

from Eq. (2) we obtain

$$
\begin{aligned}
\left\{\frac{d^{2}}{d x^{2}}\right. & +f_{1}(\pi(x) ; h(x)) \frac{d}{d x}+f_{2}(\pi(x) ; h(x)) \\
& \left.-2\left[h^{\prime}(x)\right]^{2}\left[U(h(x))-\epsilon_{n}\right]\right\} \psi_{n}(x)=0,
\end{aligned}
$$


where

$$
\begin{aligned}
& f_{1}(\pi(x) ; h(x))=2 \pi^{\prime}(x)\left[\frac{1}{\pi(x)}-\frac{h^{\prime \prime}(x)}{\pi^{\prime}(x) h^{\prime}(x)}\right] \\
& f_{2}(\pi(x) ; h(x))=\pi^{\prime \prime}(x)\left[\frac{1}{\pi(x)}-\frac{h^{\prime \prime}(x) \pi^{\prime}(x)}{\pi(x) \pi^{\prime \prime}(x) h^{\prime}(x)}\right]
\end{aligned}
$$

and prime denotes the derivative to the spatial coordinate. If the following equalities are satisfied,

$$
\begin{aligned}
h^{\prime}(x) & =m(x) \pi^{2}(x), \\
V(x)-E_{n} & =\frac{\left[h^{\prime}(x)\right]^{2}}{m(x)}\left[U(h(x))-\epsilon_{n}\right]+f(m(x) ; h(x)),
\end{aligned}
$$

then the transformation given in Eqs. (3) and (4) is a point canonical transformation. ${ }^{[2]}$ The function $f(m(x) ; h(x))$ in the above equation is given

$$
f(m(x) ; h(x))=\frac{1}{4 m(x)}\left[\frac{m^{\prime \prime}(x)}{m(x)}-\frac{3}{2}\left(\frac{m^{\prime}(x)}{m(x)}\right)^{2}-\frac{h^{\prime \prime \prime}(x)}{h^{\prime}(x)}+\frac{3}{2}\left(\frac{h^{\prime \prime}(x)}{h^{\prime}(x)}\right)^{2}\right] .
$$

Taking $h^{\prime}(x)=\sqrt{m(x)}$ and defining the parameter $\sigma(x)$ as $\sigma(x)=\frac{1}{\beta} \int h^{\prime}(x) d x$, we obtain the energy spectrum, the corresponding wave functions, and the target potential as

$$
\begin{aligned}
E_{n} & =\epsilon_{n} \\
\phi_{n}(x) & =\sqrt{\frac{h^{\prime}(x)}{m(x)}} \psi_{n}(\beta \sigma(x)), \\
V(x) & =U(\beta \sigma(x))+\frac{1}{4 m(x)}\left[\frac{1}{2} \frac{m^{\prime \prime}(x)}{m(x)}-\frac{7}{8}\left(\frac{m^{\prime}(x)}{m(x)}\right)^{2}\right] .
\end{aligned}
$$

where the parameter $\beta$ is the scale parameter. ${ }^{[2]}$

Giving a PDM function and taking an exactly solvable reference potential $U(y)$, we construct a PCT defined by Eqs. (3) and (4) and obtain a target potential, which can be used to solve the effective mass SE exactly, eigenvalues, and wave functions of the potential.

Now, let us look the case of the radial effective-mass SE, which can be written as ${ }^{[2]}$

$$
\left\{\frac{1}{2 m(r)} \frac{d^{2}}{d r^{2}}-\frac{\ell(\ell+1)}{2 m(r) r^{2}}+\frac{m^{\prime}(r)}{2 m^{2}(r)}\left(\frac{1}{r}-\frac{d}{d r}\right)-V(r)\right\} \phi_{n \ell}(r)=-E_{n \ell} \phi_{n \ell}(r),
$$

where $\ell$ is the angular-momentum quantum number and the radial wave function is taken as $\Psi_{n \ell}(r)=\phi_{n \ell}(r) / r$.

The radial $\mathrm{SE}$ for the case of constant mass reads

$$
\left\{\frac{1}{2} \frac{d^{2}}{d \rho^{2}}-\frac{\ell^{\prime}\left(\ell^{\prime}+1\right)}{\rho^{2}}-U(\rho)\right\} \psi_{n \ell^{\prime}}(r)=-\epsilon_{n \ell^{\prime}} \psi_{n \ell^{\prime}}(r) .
$$


where $\ell^{\prime}$ is the angular-momentum. By using the transformations

$$
\begin{aligned}
\rho & =h(r), \\
\psi_{n \ell^{\prime}}(r) & =\pi(r) \phi_{n \ell}(r) .
\end{aligned}
$$

from Eq. (15) we obtain

$$
\begin{aligned}
\left\{\frac{d^{2}}{d r^{2}}\right. & +f_{1}(\pi(r) ; h(r)) \frac{d}{d r}+f_{2}(\pi(r) ; h(r))-\ell^{\prime}\left(\ell^{\prime}+1\right)\left(\frac{h^{\prime}(r)}{h(r)}\right)^{2} \\
& \left.-2\left[h^{\prime}(r)\right]^{2}\left[U(h(r))-\epsilon_{n \ell^{\prime}}\right]\right\} \psi_{n \ell^{\prime}}(r)=0
\end{aligned}
$$

Comparing with Eq. (14) gives $\pi(r)=\sqrt{h^{\prime}(r) / m(r)}$ and the following

$$
\begin{aligned}
V(r)-E_{n \ell}+\frac{\ell(\ell+1)}{2 m(r) r^{2}} & =\frac{\left[h^{\prime}(r)\right]^{2}}{m(r)}\left[U(h(r))-\epsilon_{n \ell^{\prime}}\right]+\frac{\ell^{\prime}\left(\ell^{\prime}+1\right)}{2 m(r)}\left(\frac{h^{\prime}(r)}{h(r)}\right)^{2} \\
& +\frac{m^{\prime}(r)}{2 m^{2}(r) r}+f(m(r) ; h(r)) .
\end{aligned}
$$

which gives the target potential $V(r)$ and its energy eigenvalues $E_{n \ell}$ for a given radial effective mass function $m(r){ }^{[2]}$

\section{A) Kratzer potential.}

We consider the following form of the one-dimensional Kratzer potential ${ }^{[19]}$

$$
U(y)=\frac{A}{y}+\frac{B}{y^{2}},
$$

which has been extensively used to explain the molecular structure and interactions in quantum and molecular chemistry. ${ }^{[20]}$ The energy eigenvalues and the corresponding wave functions of the reference potential $U(y) \operatorname{are}^{[21]}$

$$
\begin{aligned}
\epsilon_{n \ell^{\prime}} & =-\frac{2 A^{2}}{[2 n+1+\sqrt{1-16 B}]^{2}}, \\
\psi_{n \ell^{\prime}}(r) & =a_{n} y^{\frac{1}{2}+\sqrt{-A}} e^{-\xi y}{ }_{1} F_{1}(-n ; 1+2 \sqrt{-A} ; 2 \xi y) ; \xi=\sqrt{-2 \epsilon_{n \ell^{\prime}}},
\end{aligned}
$$

where ${ }_{1} F_{1}(-n ; \varrho ; x)$ are the hypergeometric type function. Now, we give the explicit form of the target potentials and its wave functions for the following three different mass distributions.

(1) Mass function $m(x)=\left(\delta+x^{2}\right)^{2} /\left(1+x^{2}\right)^{2}$ We obtain $\sigma(x)$ as $\sigma(x)=(1 / \tau)[x+$ $(\delta-1) / \tan x]$ and the target potential from Eq. $(13)$

$$
V(x)=\frac{\theta \beta}{\left[x+\frac{\delta-1}{\tan x}\right]}\left[1+\frac{\theta \beta}{x+\frac{\delta-1}{\tan x}}\right]+\frac{\delta-1}{2} \frac{3 x^{4}+2(2-\delta) x^{2}-\delta}{\left(\delta+x^{2}\right)^{4}},
$$


where $\theta=A / \beta$ and we have set the potential parameter $B$ as $B=A^{2}$. The energy spectrum and the corresponding wave functions are written from Eqs. (11) and (12) as

$$
\begin{aligned}
E_{n} & =-\frac{\theta^{2} \beta^{2}}{\left[2 n+1+\sqrt{1-16 \theta^{2} \beta^{2}}\right]^{2}}, \\
\phi_{n}(x) & =a_{n} \sqrt{\frac{\delta+x^{2}}{1+x^{2}}}[\beta \sigma(x)]^{\frac{1}{2}+\sqrt{-A}} \exp [-\xi \beta \sigma(x)]_{1} F_{1}(-n ; 1+2 \sqrt{-A} ; 2 \xi \beta \sigma(x)) .
\end{aligned}
$$

(2) Mass function $m(x)=a /\left(\delta^{\prime}+x^{2}\right)$ The distribution corresponds to an asymptotically vanishing mass function. It gives $\sigma(x)=(\sqrt{a} / \beta) \ln \left[x+\sqrt{1+x^{2}}\right]$. Therefore, we obtain the target potential

$$
V(x)=\frac{\theta \beta}{\sqrt{a} \ln \left[x+\sqrt{1+x^{2}}\right]}\left[1+\frac{\theta \beta}{\sqrt{a} \ln \left[x+\sqrt{1+x^{2}}\right]}\right]-\frac{x^{2}+2 \delta^{\prime}}{8 a\left(\delta^{\prime}+x^{2}\right)},
$$

and the energy spectra and their wave functions are

$$
\begin{aligned}
E_{n} & =-\frac{\theta^{2} \beta^{2}}{\left[2 n+1+\sqrt{1-16 \theta^{2} \beta^{2}}\right]^{2}}, \\
\phi_{n}(x) & =\frac{A_{n}}{\left(\delta^{\prime}+x^{2}\right)^{1 / 4}}[\beta \sigma(x)]^{\frac{1}{2}+\sqrt{-A}} \exp [-\xi \beta \sigma(x)]_{1} F_{1}(-n ; 1+2 \sqrt{-A} ; 2 \xi \beta \sigma(x)) .
\end{aligned}
$$

where the normalization constant is $A_{n}=a^{1 / 4} a_{n}$.

(3) Mass function $m(x)=1+\tanh \left(\delta^{\prime \prime} x\right)$ We obtain the following target potential, energy eigenvalues and the corresponding eigenfunctions for this case,

$$
\begin{aligned}
V(x) & =\theta[\sigma(x)]^{-1}\left[1+\theta[\sigma(x)]^{-1}\right]-\frac{\left(\delta^{\prime \prime}\right)^{2}}{32} \frac{7+\tanh \left(\delta^{\prime \prime} x\right)}{\left[\sinh \left(\delta^{\prime \prime} x\right)+\cosh \left(\delta^{\prime \prime} x\right)\right]^{2}}, \\
E_{n} & =-\frac{\theta^{2} \beta^{2}}{\left[2 n+1+\sqrt{1-16 \theta^{2} \beta^{2}}\right]^{2}}, \\
\phi_{n}(x) & =a_{n}\left[1+\tanh \left(\delta^{\prime \prime} x\right)\right]^{1 / 4}[\beta \sigma(x)]^{\frac{1}{2}+\sqrt{-A}} \\
& \times \exp [-\xi \beta \sigma(x)]_{1} F_{1}(-n ; 1+2 \sqrt{-A} ; 2 \xi \beta \sigma(x)),
\end{aligned}
$$

where $\sigma(x)=\left[\sqrt{2} /\left(\beta \delta^{\prime \prime}\right)\right] \tanh ^{-1}\left[\sqrt{1+\tanh \left(\delta^{\prime \prime} x\right)} / \sqrt{2}\right]$. It is seen that the Schrdinger equation can be exactly solved by using point canonical transformation in one-dimension.

(B) Family of power law plus inverse power potentials. We study the target potential and its energy eigenvalues, and wave functions for the following reference potentials in the form $U(\rho)=-\frac{a}{\rho}+c \rho^{p}(p=0,-2)$ and $U(\rho)=\frac{a}{\rho^{2}}+c \rho^{2}$ for the radial SE for the case of position dependent mass.

(1) $U(\rho)=-\frac{a}{\rho}+c$. The reference potential has the form

$$
U(\rho)=-\frac{a}{\rho}+c
$$


which describes a hydrogen atom with the energy eigenvalue $E-c$ having the energy eigenvalues and corresponding wave functions,

$$
\begin{aligned}
\epsilon_{n \ell^{\prime}} & =c-\frac{a^{2}}{2}\left(n+\ell^{\prime}+1\right)^{-2}, \\
\psi_{n \ell^{\prime}}(\rho) & =a_{n} \rho^{\ell^{\prime}+1} \exp \left[-\frac{a}{n+\ell^{\prime}+1} \rho\right]{ }_{1} F_{1}\left(-n ; 2 \ell^{\prime}+2 ; \frac{2 a}{n+\ell^{\prime}+1} \rho\right) .
\end{aligned}
$$

Taking the radial mass function $m(r)=\mu r^{\kappa}$ and the PCT function $h(r)=r^{\nu}$ and inserting into Eq. (19), we obtain

$$
\begin{aligned}
V(r)-E_{n \ell}+\frac{\ell(\ell+1)}{2 \mu} r^{-\kappa-2} & =-\frac{a \nu^{2}}{\mu} r^{\nu-\kappa-2}-\frac{\nu^{2} \epsilon_{n \ell^{\prime}}}{\mu} r^{2 \nu-\kappa-2} \\
& +\frac{r^{-\kappa-2}}{2 \mu}\left[\ell^{\prime}\left(\ell^{\prime}+1\right) \nu^{2}+\kappa+\frac{1}{2} \kappa(\kappa-1)\right. \\
& \left.-\frac{3}{4} \kappa^{2}-\frac{1}{2}(\nu-1)(\nu-2)+\frac{3}{4}(\nu-1)^{2}\right],
\end{aligned}
$$

which gives two possible solutions

(a) For the case of $\nu=1+\frac{\kappa}{2} ; \kappa \neq-2$, from Eq. (35) we obtain

$$
\begin{aligned}
V(r) & =-\mu C^{2} r^{-1-\frac{\kappa}{2}} \\
E_{n \ell} & =-\frac{1}{2} a \mu C^{2}\left(n+|\mathcal{L}(\ell)|+\frac{1}{2}\right)^{-2}
\end{aligned}
$$

where $|\mathcal{L}(\ell)|=\mp \frac{1}{\kappa+2} \sqrt{4 \ell(\ell+1)+(\kappa-1)^{2}}$ and $C$ is a real coupling parameter. From Eq. (17) we obtain corresponding wave functions

$$
\begin{aligned}
\phi_{n \ell}(r) & =b_{n} \sqrt{\frac{2 \mu}{\kappa+2}} \exp \left[-\zeta r^{1+\frac{\kappa}{2}}\right] r^{\left(1+\frac{\kappa}{2}\right)\left(|\mathcal{L}(\ell)|+\frac{1}{2}\right)+\frac{\kappa}{4}} \\
& \times{ }_{1} F_{1}\left(-n ; 2|\mathcal{L}(\ell)|+1 ; 2 \zeta r^{1+\frac{\kappa}{2}}\right)
\end{aligned}
$$

where $\zeta=a^{\prime} /\left(n+|\mathcal{L}(\ell)|+\frac{1}{2}\right)$, and $b_{n}$ is a normalization constant.

(b) For the case of $\nu=\kappa+2 ; \kappa \neq-2$, following the above procedure, we obtain the target potential, the eigenvalues and the eigenfunctions by taking into account the independence of a potential from any quantum number,

$$
\begin{gathered}
V(r)=-\frac{1}{2} \mu C r^{\kappa+2}, \\
E_{n \ell}=\frac{\mu C}{2 a}(2 n+2 \mathcal{L}(\ell)+1)^{2}, \\
\phi_{n \ell}(r)=b_{n} \sqrt{\frac{\mu}{\kappa+2}} \exp \left[-\zeta r^{\kappa+2}\right] r^{(\kappa+2)\left(|\mathcal{L}(\ell)|+\frac{1}{2}\right)-\frac{1}{2}} \\
\times{ }_{1} F_{1}\left(-n ; 2|\mathcal{L}(\ell)|+1 ; 2 \zeta r^{1+\frac{\kappa}{2}}\right),
\end{gathered}
$$


where $b_{n}$ is a normalization constant and $C$ is the coupling parameter.$^{[2]}$

The particular case $\kappa=-2$ is solved by taking $h(r)=\frac{1}{a} \ln (r)^{[2]}$ to obtain the target potential. In that case the energy eigenvalues, and the corresponding wave functions only exist for the ground state. ${ }^{[2]}$ We summarize the potential and the energy eigenvalues by using Eq. (19)

$$
\begin{aligned}
V(r) & =-\frac{1}{\mu l n(r)}+\frac{1}{2} C \ln ^{-2}(r), \\
E_{n \ell} & =\frac{1}{\mu}\left\{-\frac{1}{2}\left[n+\frac{1}{2}+|\Lambda(\ell)|\right]^{-2}-\frac{9}{8}\right\},
\end{aligned}
$$

where $|\Lambda(\ell)|= \pm \frac{1}{2} \sqrt{1+4 \mu C}$. The corresponding wave functions are

$$
\phi_{n \ell}(r)=b_{n} r^{-1 / 2}[\ln (r)]^{\frac{1}{2}+|\Lambda(\ell)|} \exp [-\eta \ln (r)]_{1} F_{1}(-n ; 1+2|\Lambda(\ell)| ; 2 \eta \ln (r)),
$$

where $\eta=\left(n+\frac{1}{2}+|\Lambda(\ell)|\right)^{-1}$ and $b_{n}=\sqrt{\mu}\left(a^{\prime}\right)^{-|\Lambda(\ell)|}$.

(2) $U(\rho)=-\frac{a}{\rho}+\frac{c}{\rho^{2}}$. The reference potential has the form

$$
U(\rho)=-\frac{1}{\rho}\left(a+\frac{c}{\rho}\right),
$$

and the energy eigenvalues and the corresponding wave functions are listed as ${ }^{[22]}$

$$
\begin{aligned}
\epsilon_{n \ell^{\prime}} & =-\frac{a^{2}}{2}\left(n+\frac{1}{2}+\sqrt{\left(\ell^{\prime}+\frac{1}{2}\right)^{2}+2 c}\right)^{-2}, \\
\psi_{n \ell^{\prime}}(\rho) & =a_{n} \exp \left[-\frac{a}{n+\Lambda+1} \rho\right]_{1} F_{1}\left(-n ; 2 \Lambda+2 ; \frac{2 a}{n+\Lambda+1} \rho\right),
\end{aligned}
$$

where $\Lambda=-\frac{1}{2}+\sqrt{\left(\ell^{\prime}+\frac{1}{2}\right)^{2}+2 c}$. Following the same procedure, we can write the results of two possible solutions

(a) For the case of $\nu=1+\frac{\kappa}{2} ; \kappa \neq-2$, the target potential and its energy spectrum are written as

$$
\begin{aligned}
V(r) & =-\frac{a \gamma^{2}}{\mu} r^{-1-\frac{\kappa}{2}} \\
E_{n \ell} & =-\frac{a^{2} \gamma^{2}}{2 \mu}\left(n+\frac{1}{2}+\sqrt{|\mathcal{L}(\ell)|^{2}+2 c}\right)^{-2},
\end{aligned}
$$

where $\mathcal{L}(\ell)=\mp \frac{1}{\kappa+2} \sqrt{4 \ell(\ell+1)+(\kappa-1)^{2}-2 c(\kappa+2)^{2}}$ and the corresponding wave functions read

$$
\begin{aligned}
\phi_{n \ell}(r) & =b_{n} \sqrt{\frac{2 \mu}{\kappa+2}} r^{\kappa / 4} \exp \left[-\frac{a}{n+\frac{1}{2}+\sqrt{|\mathcal{L}(\ell)|^{2}+2 c}} r^{1+\frac{\kappa}{2}}\right] \\
& \times{ }_{1} F_{1}\left(-n ; 1+2 \sqrt{|\mathcal{L}(\ell)|^{2}+2 c} ; \frac{2 a}{n+\frac{1}{2}+\sqrt{|\mathcal{L}(\ell)|^{2}+2 c}} r^{1+\frac{\kappa}{2}}\right) .
\end{aligned}
$$


where $b_{n}$ is a normalization constant.

(b)For the case of $\nu=\kappa+2 ; \kappa \neq-2$, we list the target potential and the energy spectrum keeping in mind that any potential function must be independent from any quantum number

$$
\begin{aligned}
V(r) & =-2 \mu C r^{\kappa+2}, \\
E_{n \ell} & =-\frac{4 \mu C}{a} \frac{1}{\left(n+\frac{1}{2}+\sqrt{|\mathcal{L}(\ell)|^{2}+2 c}\right)^{-2}},
\end{aligned}
$$

where $\mathcal{L}(\ell)$ is given in $(a)$. The corresponding wave functions are written as

$$
\begin{aligned}
\phi_{n \ell}(r) & =b_{n} \sqrt{\frac{\mu}{\kappa+2}} r^{-1 / 2} \exp \left[-\frac{a}{n+\frac{1}{2}+\sqrt{|\mathcal{L}(\ell)|^{2}+2 c}}\right] \\
& \times{ }_{1} F_{1}\left(-n ; 1+2 \sqrt{|\mathcal{L}(\ell)|^{2}+2 c} ; \frac{2 a}{n+\frac{1}{2}+\sqrt{|\mathcal{L}(\ell)|^{2}+2 c}} r^{\kappa+2}\right) .
\end{aligned}
$$

Finally, we study the particular case where $\kappa=-2$ by taking $h(r)=\frac{1}{a} \ln (r)$. By using Eq. (19), we summarize the target potential and its energy eigenvalues as

$$
\begin{aligned}
V(r) & =-\frac{1}{\mu \ln (r)}+\frac{1}{\ln ^{2}(r)}\left[\frac{c}{\mu}+\frac{C}{2}\right], \\
E_{n \ell} & =\frac{1}{\mu}\left\{-\frac{1}{2}\left[n+\frac{1}{2}+\sqrt{\left|\Lambda^{\prime}(\ell)\right|+2 c}\right]^{2}-\frac{9}{8}\right\},
\end{aligned}
$$

where $\left|\Lambda^{\prime}(\ell)\right|= \pm \frac{1}{2} \sqrt{1+4 \mu C}$. The corresponding wave functions are

$$
\phi_{n \ell}(r)=b_{n} r^{-1 / 2} \exp \left[-\eta^{\prime} \ln (r)\right]_{1} F_{1}\left(-n ; 1+2 \sqrt{\left|\Lambda^{\prime}(\ell)\right|^{2}+2 c} ; 2 \eta^{\prime} \ln (r)\right),
$$

where $\eta^{\prime}=\left(n+\frac{1}{2}+\sqrt{\left|\Lambda^{\prime}(\ell)\right|^{2}+2 c}\right)^{-1}$ and $b_{n}=\sqrt{a \mu}$.

(3) $U(\rho)=\frac{a}{\rho^{2}}+c \rho^{2}$. The energy eigenvalues and corresponding wave functions of this potential can be written as ${ }^{[23]}$

$$
\begin{aligned}
\epsilon_{n \ell^{\prime}} & =\sqrt{2 c}\left[2 n+1+\frac{1}{2} \sqrt{\left(2 \ell^{\prime}+1\right)^{2}+8 a}\right] \\
\psi_{n \ell^{\prime}}(\rho) & =(2 c)^{\delta / 2} \rho^{2 \delta} \exp \left[-\frac{c}{2} \rho^{2}\right]{ }_{1} F_{1}\left(-n, 2 \delta+\frac{1}{2} ; \sqrt{2 c} \rho^{2}\right) .
\end{aligned}
$$

where $\delta=\frac{1}{4}\left[1+\sqrt{\left(2 \ell^{\prime}+1\right)^{2}+8 a}\right]$. In this case, we summarize the two possible solutions as

(a) For the case of $\nu=1+\frac{\kappa}{2} ; \kappa \neq-2$,

$$
\begin{aligned}
V(r) & =\frac{c \nu^{2}}{\mu} r^{\kappa+2} \\
E_{n \ell} & =\sqrt{\frac{2 c \nu^{4}}{\mu^{2}}}\left(2 n+1+\sqrt{2|\mathcal{L}(\ell)|^{2}+2 a}\right),
\end{aligned}
$$


where $\mathcal{L}(\ell)=\mp \frac{1}{\kappa+2} \sqrt{4 \ell(\ell+1)+(\kappa-1)^{2}-2 a(\kappa+2)^{2}}$, and the corresponding wave functions

$$
\phi_{n \ell}(r)=b_{n}^{\prime} \sqrt{\frac{2 \mu}{\kappa+2}} r^{\delta(\kappa+2)+\frac{\kappa}{4}} \exp \left[-\sqrt{\frac{c}{2}} r^{\kappa+2}\right]{ }_{1} F_{1}\left(-n, 2 \delta+\frac{1}{2} ; \sqrt{2 c} r^{\kappa+2}\right),
$$

where $b_{n}^{\prime}=b_{n}(2 c)^{\delta / 2}$ and $b_{n}$ is a normalization constant.

(b) For the case of $\nu=\frac{1}{2}+\frac{\kappa}{4} ; \kappa \neq-2$, we give the target potential and its energy eigenvalues as

$$
\begin{aligned}
V(r) & =-2 \mu C r^{-\frac{\kappa}{2}-1} \\
E_{n \ell} & =-\sqrt{2 C^{2} \mu^{2} c} \frac{1}{2 n+1+\sqrt{\left|\mathcal{L}^{\prime}(\ell)\right|^{2}+2 a}},
\end{aligned}
$$

where $\mathcal{L}^{\prime}(\ell)=\mp \frac{1}{\kappa+2} \sqrt{16 \ell(\ell+1)+4(\kappa-1)^{2}-2 a(\kappa+2)^{2}}$. The corresponding wave functions are written as

$$
\phi_{n \ell}(r)=b_{n}^{\prime} \sqrt{\frac{2 \mu}{\kappa+2}} r^{\delta\left(1+\frac{\kappa}{2}\right)+\frac{\kappa}{4}} \exp \left[-\sqrt{\frac{c}{2}} r^{1+\frac{\kappa}{2}}\right]{ }_{1} F_{1}\left(-n, 2 \delta+\frac{1}{2} ; \sqrt{2 c} r^{\frac{\kappa}{2}}\right) .
$$

Now, we proceed the particular case where $\kappa=-2$ as a last situation by taking $h(r)=$ $\frac{1}{a} \ln (r)$. Following the same procedure gives

$$
\begin{aligned}
V(r) & =\frac{c}{\mu a^{4}} \ln ^{2}(r)+\frac{1}{\ln ^{2}(r)}\left(\frac{a}{\mu}+\Theta\right), \\
E_{n \ell} & =\sqrt{\frac{2 c}{\mu^{2} a^{4}}}\left(2 n+1+\sqrt{|\Gamma(\ell)|^{2}+2 a}\right)-\frac{9}{8 \mu} .
\end{aligned}
$$

where $\Gamma(\ell)= \pm \frac{1}{2} \sqrt{1+8 \mu \Theta}$ and $\Theta$ is a real coupling parameter. ${ }^{[2]}$ The wave functions can be given as

$$
\phi_{n \ell}(r)=b_{n} \sqrt{\mu a}\left(\frac{\sqrt{2 c}}{a^{2}}\right)^{\delta} r^{-(1 / 2)} \exp \left[-\sqrt{\frac{c}{2 a^{4}}} \ln ^{2}(r)\right]{ }_{1} F_{1}\left(-n, 2 \delta+\frac{1}{2} ; \sqrt{\frac{2 c}{a^{4}}} \ln ^{2}(r)\right),
$$

where $\delta=\frac{1}{4}\left[1+\sqrt{4|\Gamma(\ell)|^{2}+8 a}\right]$.

In summary, we have used the PCT in the position-dependent mass formalism and choose the Kratzer-like potential in one-dimension as the reference potential to construct the exact solvable target potential and write the energy eigenvalues, and the corresponding wave functions in closed forms. We apply the extended formalism of the PCT to the threedimensional problems and use the potentials $V(\rho)=-\frac{a}{\rho}+c \rho^{p}(p=0,-2)$ and $V(\rho)=$ $\frac{a}{\rho^{2}}+c \rho^{2}$ as the reference potentials. The results show that a suitable transformation can 
be used to convert the wave equation into the exact solvable SE and therefore the required energy eigenvalues and wave functions are obtained exactly.

[1] Gendenstein L 1983 JETP Lett. 38356

[2] Alhaidari A D 2002 Phys. Rev. A 66042116

[3] De R, Dutt R and Sukhatme U 1992 J. Phys. A 25 L843

[4] Dutt R, Khare A and Varshni Y P 1995 J. Phys. A 28 L107

[5] Jia C S, Diao Y F, Li M, Yang Q B, Sun L T and Huang R Y 2004 J. Phys. A 3711275

[6] Alhaidari A D 2001 Phys. Rev. Lett. 87210405

[7] Roy B and Roy P 2002 J. Phys. A 353961

[8] Dutra A S and Almeida C A S 2000 Phys. Lett. A 27525

[9] Plastino A R, Rigo A, Casas M, Gracias F and Plastino A 1999 Phys. Rev. A 604318

[10] Alhaidari A D 2003 Int. J. Theor. Phys. 422999

[11] Yu J and Dong S H 2004 Phys. Lett. A 325194

[12] Jiang L, Yi L Z and Jia C S 2005 Phys. Lett. A 345279

[13] Tezcan C and Sever R 2008 Int. J. Theor. Phys. 471471

[14] Serra L and Lipparini E 1997 Europhys. Lett. 40667

[15] von Roos O 1983 Phys. Rev. B 277547

[16] von Roos O and Mavromatis H 1985 Phys. Rev. B 312254

[17] de Saavedra F A, Boronat J, Polls A and Fabrocini A 1994 Phys. Rev. B 504248

[18] Barranco M, Pi M, Gatica S M, Hernandez E S and Navarro J 1997 Phys. Rev. B 568997

[19] Kratzer A 1920 Z. Phys. 3289

[20] Roy R J L and Bernstein R B 1970 J. Chem. Phys. 523869

[21] Maharana K, [arXiv: math-ph/0401026

[22] Aygun M, Bayrak O and Boztosun I 2007 J. Phys. B: At. Mol. Opt. Phys. 405372

[23] Constantinescu F and Magyari E 1971 Problems in Quantum Mechanics (Oxford: Pergamon.) 